\title{
DENTIN HYPERSENSITIVITY - REVISITED
}

Pradnya V. Bansode1, Seema D. Pathak ${ }^{2}$, Savita P. Wagh ${ }^{3}$

\section{HOW TO CITE THIS ARTICLE:}

Pradnya V Bansode, Seema D Pathak, Savita P Wagh. "Dentin hypersensitivity - revisited". Journal of Evolution of Medical and Dental Sciences 2013; Vol2, Issue 34, August 26; Page: 6466-6473.

ABSTRACT: Hypersensitive tooth is an old age complaint. It can be defined as pain arising from exposed dentin typically in response to chemical, thermal or osmotic stimuli that cannot be explained as arising from any other form of dental defect or pathology. This enigma of hypersensitivity commonly affects buccal cervical areas of premolars \& canines. It affects the population at an age range of 20-40 yrs with peak at end of third decade. Sensitivity occurs when dentinal tubules are exposed. Four theories have been proposed to explain this mechanism, which are transducer, modulation, gate control and hydrodynamic. Hydrodynamic theory is widely accepted. Old remedies and materials used for management of hypersensitivity includes lung ash from mice, dried livers from lizards, salt lavage, potassium nitrate, alum, anodynes such as cocaine hydrochloride, menthol, phenol, eugenol, morphine or codeine sulfate. Caustics such as zinc chloride, absolute alcohol, trichloroacetic acid, silver nitrate and others such as carbolic acid, calcium compounds such as calcium hydroxide, dicalcium phosphate, oxalates. Stannous or sodium fluoride, fluoride iontophoresis or leukomscy's paste, glass ionomers, resins, cyanoacrylates and corticosteroids. Recent methods include dentin treatment by lasers, CCP- ACP, Pro-Argin technology. KEYWORDS: Dentinal tubules, hypersensitive dentin, hydrodynamic theory, fluorides.

INTRODUCTION: Hypersensitive tooth is an age old complaint of a man. It can be defined as "pain arising from exposed dentin typically in response to chemical, thermal or osmotic stimuli that cannot be explained as arising from any other form of dental defect or pathology".1

Many patients avoid dental treatment because of the hypersensitivity. Surprisingly, most practices do not have a systematic approach for diagnosis and treating this condition. This is simply because it seems to be complicated. Many practitioners have had poor success in the past with sensitivity treatments and are reluctant to try it again. Today's products are effective and easy to use. This enigma of hypersensitivity commonly affects buccal cervical areas of first premolars and less frequently canines, second premolars, incisors and molars (fig 1). It mainly affects the population at an age range of 20-40 yrs with peak at the end of third decade. ${ }^{1}$

ETIOLOGY: The factors predispose the patient to the essential components of dentin hypersensitivity: exposed, open and patent dentinal tubules leading to a vital pulp (fig 2).There may also be passage of fluids through the enamel. The enamel may be thought of as a semipermeable membrane that allows passage of fluids and small molecules through the organic defects between the enamel crystals. With time, the organic channels become plugged due to the formation of organic biofilm (fig 3). When this occurs, the bi-directional flow of fluids stops and so does the pain.

Histochemical, autoradiographic and electron microscopic studies have vividly described the relationship between odontoblast and neural fibers of the pulp but exact mechanism of dentin hypersensitivity is only hypothesized. ${ }^{2}$ Four theories have been proposed to explain this mechanism of dentin sensitivity, which are transducer, modulation, gate control and hydrodynamic. 
Hydrodynamic theory is currently a widely accepted theory. Hydrodynamic theory states that dentinal pain results from a stimulus causing minor changes in the fluid movement within the dentinal tubules (fig 4); this may subsequently deform the odontoblast or its process and cause an elicitation of pain via the intimately associated mechanoreceptor like nerve endings.

TREATMENT: The first line of treatment for dentin hypersensitivity is of course prevention. The predisposing factors must be dealt with first. Periodontal disease, recession, occlusal forces and diet present many challenges.

To overcome the enigma of hypersensitive dentin several possible approaches have been proposed such as:

1. Sealing or physically occluding the outer end of the dentinal tubules.

2. Coagulating the tubular protoplasm by chemical treatment.

3. Providing a chemical ion that can precipitate one of the components of protoplasmic fluid and thus create a tubular plug.

4. Providing an agent that will permeate through the dentinal tubules and suppress the firing of the nerves of the dental pulp.(fig 5)

Old remedies and materials since decades used for management of hypersensitive dentin includes use of lung ash from mice, dried livers from lizards and salt lavage, potassium nitrate, alum, anodynes such as cocaine hydrochloride, clove oil, menthol, phenol, eugenol morphine sulfate or codeine sulfate. Caustics such as zinc chloride, absolute alcohol, trichloroacetic acid, silver nitrate and other materials such as carbolic acid, calcium compounds such as calcium hydroxide, dicalcium phosphate etc. and oxalates. Fluoride such as stannous fluoride, sodium fluoride and fluoride iontophoresis, glass ionomers resins, cyanoacrylates and corticosteroids. Recent methods include dentin treatment by lasers, CPP- ACP and Pro-Argin technology. Wide range of products are available for treatment. The product must either block the movements of fluid in the tubules or stop the transmission of the pain response to the pulp. Different treatments may be tried and modified based on the patient's response. The first group of products works by occluding the open tubules and decreasing pulpal fluid flow. This group includes: fluorides, fluoride varnishes tissue fixatives, oxalates, remineralising agents, and Pro-Argin technology. The second group of products works by depolarizing the nerve so that it cannot transmit the pain response.

The hypersensitive dentin can be treated by:

i. $\quad$ Occluding the open dentinal tubules.

ii. Depolarization of the nerve fibres.

\section{I.}

OCCLUSION OF DENTINAL TUBULES

Fluorides: Patients can apply stannous fluoride in a $0.4 \%$ gel or sodium fluoride in a $0.5 \%$ mouth rinse or a $1.1 \%$ gel. Fluorides reduce the permeability of dentin probably by precipitation of insoluble calcium fluoride inside the dentinal tubules and reduce sensitivity. Fluoride application is believed to work through a reaction between the fluoride ion and ionized calcium in the tubular fluid. This reaction forms an insoluble calcium fluoride precipitate in the tubules. ${ }^{4}$ Different fluoride show different efficacies. Stannous fluoride is more effective than sodium fluoride in concentrations used for toothpaste formulations. 
Fluoride varnishes: Fluoride varnishes may be used for sensitivity relief but are chiefly indicated for caries control and remineralisation. The desensitization effect is transient since the material is abraded soon after placement. Many applications may be necessary for increased efficacy. It is thought that the benefit comes from the physical blockage of the tubules by the varnish base than the fluoride itself. ${ }^{4}$ Duraphat (Colgate Oral Pharmaceuticals) is a $5 \%$ sodium fluoride varnish applied in the office with a syringe-style applicator at approximately 0.3 to $0.5 \mathrm{~mL}$ of varnish to each tooth, and dental floss can be used to draw the varnish interproximally. Topical fluorides are believed to form a barrier by precipitating calcium fluoride on the exposed dentin surface and reduce dentin permeability.

Fluoride iontophoresis: It is the process of influencing ionic motion by an electric current and has been used as a desensitizing procedure in conjunction with sodium fluoride. There is immediate reduction in sensitivity after treatment with iontophoresis. ${ }^{5}$

Tissue fixatives: Tissue fixatives desensitizing products contain agents such as glutaraldehyde or HEMA. These agents bind to tissue fluid proteins in the dentinal tubules and the superficial cells of the subsequent pulp and denature (coagulate) these proteins. These products cannot be placed near the gingival epithelium since they may cause necrosis of the gingival as well as the loss of the biological attachment. 6

Oxalates: Pashley and Galloway advocated use of potassium oxalate crystals to the dentinal tubules. Desensitizers containing metallic salts, predominantly oxalates, form insoluble chemical precipitates in the peritubular dentin. No acid etch or light curing is needed for oxalate use. They cause no irritation of the gingival tissues. It forms a complex with the calcium rich zone of the peritubular dentin to create a crystal plug. This effectively shuts down dentin sensitivity at near $100 \%$ levels. ${ }^{7}$

Remineralising Pastes: Remineralising Pastes are used in office or at home to restore the minerals that have leached out of patient's teeth due to caries, diet etc. these pastes have the added advantage of reducing sensitivity through tubule occlusion. Two active ingredients have been shown to be most effective for this purpose: Calcium sodium phosphosilicate bioactive glass and Amorphous Calcium Phosphate. These toothpastes have been shown to significantly reduce dentin hypersensitivity, with continued home use. ${ }^{8,}{ }^{9}$ ACP-CPP is the most effective casein phosphopeptide-amorphous calcium phosphate where the casein portion (derived from milk) binds the ACP to the tooth surface \& forms a protective mineral barrier of hydroxyapatite which occludes the exposed dentinal tubules. ${ }^{10}$ These pastes are placed on the affected areas after regular brushing. They can be applied as in-office agents.

Pro-Argin Technology: In 2002, Kleinberg developed a material to reduce sensitivity based on saliva's natural role in reducing sensitivity. In healthy patients, saliva is normally very effective in reducing dentin hypersensitivity. Saliva provides calcium and phosphate which over time occlude open dentin tubules. Pro-Argin Technology was developed based on this role that saliva plays in naturally reducing hypersensitivity. ${ }^{11,12}$ The Pro-Argin formula contains arginine, an amino acid 
found in saliva. The positively charged arginine binds to the negatively charged dentin surface. This attracts calcium rich layer from the saliva to infiltrate and block the dentinal tubules. ${ }^{13}$ This technology is available for in-office application, through a paste which is delivered by prophylaxis cup. There is also toothpaste for at-home use. The in-office paste has been found to provide immediate and lasting relief of hypersensitivity for 4 weeks. ${ }^{14}$

\section{DEPOLARIZATION OF THE NERVE}

The second major group of desensitization products works by depolarizing the nerve that transmits the pain response. After the nerve is depolarized, it cannot re-polarize and this diminishes it excitability. The ingredient that produces this effect is potassium nitrate. ${ }^{15}$ According to the FDA, for potassium nitrate toothpaste to claim to be desensitizing it must contain $5 \%$ of the ingredient. Potassium nitrate penetrates the enamel and dentin to travel to the pulp and creates the calming effect on the nerve. This effect can be thought of as "anesthetic-like". ${ }^{16}$

Potassium nitrate products are Ideal for post bleaching sensitivity. Post bleaching sensitivity occurs due to easy passage of peroxide through the enamel (a semi-permeable membrane) and dentin to the pulp. Desensitization products that work by occluding the dentinal tubules are ineffective in preventing the passage of tiny peroxide molecule, which can travel in the interstitial spaces between the tubules (16). Potassium nitrate can be delivered in several effective ways to counteract post bleaching sensitivity:

\section{RECENT PROGRESS IN TREATMENT}

Laser Treatment: Laser therapy was recommended by Kimura et al ${ }^{17}$.to treat DH with effectiveness between $5.2 \%$ and $100 \%$, depending on the type of laser and parameters used. According to the authors, lasers are more effective than other treatments. The mechanism of laser treatment for DH is not well explained ${ }^{18}$, although Pashley ${ }^{19}$ suggested that it may occur through coagulation and protein precipitation of the plasma in the dentinal fluid or by alteration of the nerve fiber activity. The study by McCarthy et al. indicates that the reduction in DH could be the result of alteration of the root dentinal surface, physically occluding the dentinal tubules.

Calcium silicate: A new proposal presented by Gandolfi et al. suggests the application of a calcium silicate paste, derived from Portland cement, which is effective in tubular occlusion and reduction of dentin permeability. ${ }^{20}$

Pre-hybridized dentin: Post cementation hypersensitivity may occur after a newly cemented restoration is placed. The symptoms are usually a short, sharp pain that occurs when a tooth is exposed to thermal or chemical stimuli. Usually, post cementation hypersensitivity may occur when a tooth that has recently been restored with a crown or inlay/onlay. Prehybridized dentin or immediate dentin sealing has been suggested to make the dentin less sensitive while a restoration is fabricated in the laboratory. Because a hybrid layer is created immediately after preparation, teeth treated with the immediate dentin sealing technique were better able to tolerate thermal and functional loads in comparison to teeth that were sealed when the restorations were placed. ${ }^{21}$

Calcium phosphate precipitation: Chiang and colleagues ${ }^{21}$ found a mesoporous silica biomaterial containing nano-sized calcium oxide particles mixed with $30 \%$ phosphoric acid can occlude dentinal 
tubules and considerably reduce dentin permeability even in the presence of pulpal pressure. When the supersaturated calcium and hydrogen phosphate ion containing nano sized calcium oxide particles paste is brushed on the surface, the ions diffuse into the dentinal tubules and form dicalcium phosphate dihydrate precipitation at a depth of $100 \mathrm{~mm} .22$

CONCLUSION: There is considerable evidence that pain in teeth was treated by Chinese some 2000 yrs ago. An interest has again developed and increased as related to the tooth as pain model, physiologic cause of hypersensitivity, increased life spans, the search for better therapies and desire to identify more effective treatment for hypersensitivity.

\section{REFERENCES:}

1. Addy Martin: Etiology and clinical implication of dentin hypersensitivity. Dent Clinic North Amer. 34(3): 503-514, 1990.

2. Rosenthal MW: historic review of management of tooth hypersensitivity. Dent Clinic North Amer. 34(3): 403-427, 1990.

3. Berman Luois H: Dentinal Sensation and hypersensitivity a review of mechanism and treatment alternatives. J Periodontol. 56(4): 216-221, 1984.

4. Strassler H, Drisko C, Alexander D, Dentin hypersensitivity: its inter-relationship to gingival recession and acid erosion, Supplement to inside Dentistry, June 2008 Vol. 4:6.

5. Kern DA, McQuade, MJ et.al. Effectiveness of sodium fluoride on tooth hypersensitivity with and without iontophoresis. J. Periodontol. 1989: 60:386-389.

6. Cox, C, Physiology of dentin hypersensitivity: Clinical Treatment, Restorative \& Aesthetic Practice, Nov. 2002 Vol 4:9, 61-67.

7. Kolker JL, Vargas MA, Armstron SR, Dawson DV, Effect of dentin desensitizing agents on dentin permeability, J Dent Res (IADR Abstract \# 0295), San Diego 2000.

8. Litowski LJ, Quinlan KB ,Mc Donald NJ, Teeth hypersensitivity reduction by a novel bioglass dentifrice, J Dent Res, 1998 77:199 (Abstract \# 747).

9. Du MQ, Tai BJ, Jiang H, Zhong JP Greenspan DC, Clark AE, Efficacy of dentifrice containing bioactive glass on dentin hypersensitivity, 82nd General Session and exhibition of IADR. AAD.

10. Chalmers, J, The Evolving Technology of Amorphous Calcium Phosphate, Dimensions of Dental Hygiene Continuing Education, 4-9.

11. Panagakos, F, Schiff T, Guignon A, Dentin hypersensitivity: Effective treatment with an inoffice desensitizing paste containing $8 \%$ arginine and calcium carbonate, Am J Dent, 2009, 22( special issue) 3A-7A

12. Kleinberg I, Sensistat: A new saliva based composition for simple and effective treatment of dentinal sensitivity pain, Dent today, 2002, 42-47.

13. Strassler H, Serio, F, Dentinal Hypersensitivity Etiology, Diagnosis and Management, ineedce.com

14. Schiff T, Delgado E, Zhang YP, et.al, clinical evaluation of the efficacy of an in-office desensitizing paste containing $8 \%$ arginine and calcium carbonate and providing instant and fasting relief of dentin hypersensitivity, Am J Dent, 2009, 22(special issue) 8A-15A.

15. Handosh M, A superior desensitizer- potassium nitrate, J Am Dent Assoc, 1974, 88:831-832. 
16. Haywood V, Treating sensitivity During Tooth Whitening, Compendium, 2005 Vol. 26:9 (suppl 3) 11-20.

17. Kimura Y, Wilder-Smith P, Yonaga K, Matsumoto K (2000) Treatment of dentine hypersensitivity by lasers: a review. J ClinPeriodontol 27, 715-721. 85

18. Pashley DH (2000) Potential treatment modalities for dentine hypersensitivity: in-office products. In: Tooth wear and sensitivity: clinical advances in restorative dentistry 2000, Addy M, Embery G, Edgar WM, Orchardson R eds, Martin Dunitz, London, 351-365

19. Blaggana A.1, Vohra P.2, Nagpal A. Diagnosis and Treatment of Dentinal Hypersensitivity Journal of Innovative Dentistry, Vol 1, Issue 3, Sept-December 2011.

20. Magne $\mathrm{P}$, Kim $\mathrm{TH}$, Cascione $\mathrm{D}$, et al. Immediate dentin sealing improves bond strength of indirect restorations. J Prosthet Dent 2005; 94(6):511-9.

21. Chiang YC, Chen HJ, Liu HC, et al. A novel mesoporous biomaterial for treating dentin hypersensitivity. J Dent Res 2010; 89(3):236-40.

\section{FIGURES:}

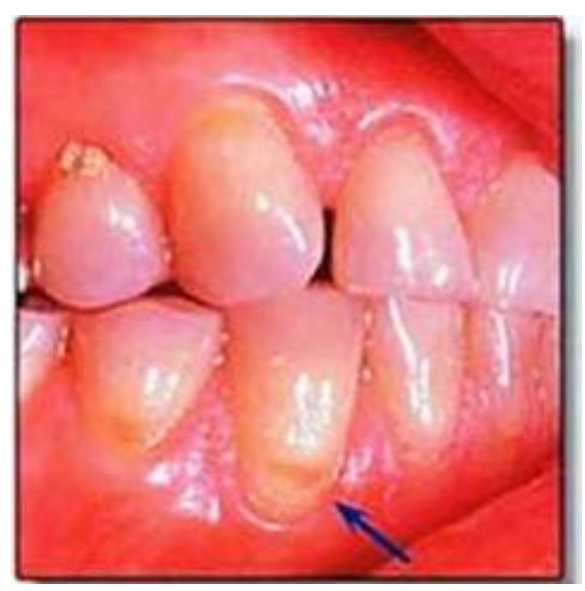

Fig. 1

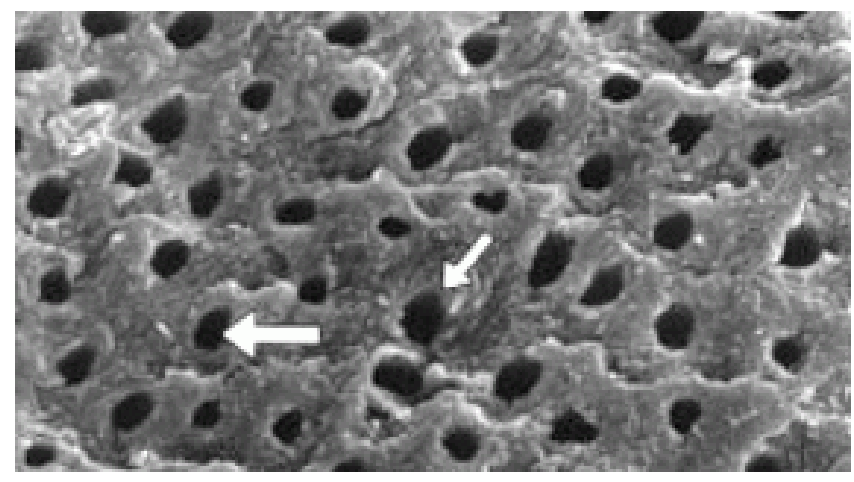

Fig. 2 


\section{REVIEW ARTICLE}

\section{HYPERSENSITIVE DENTIN}

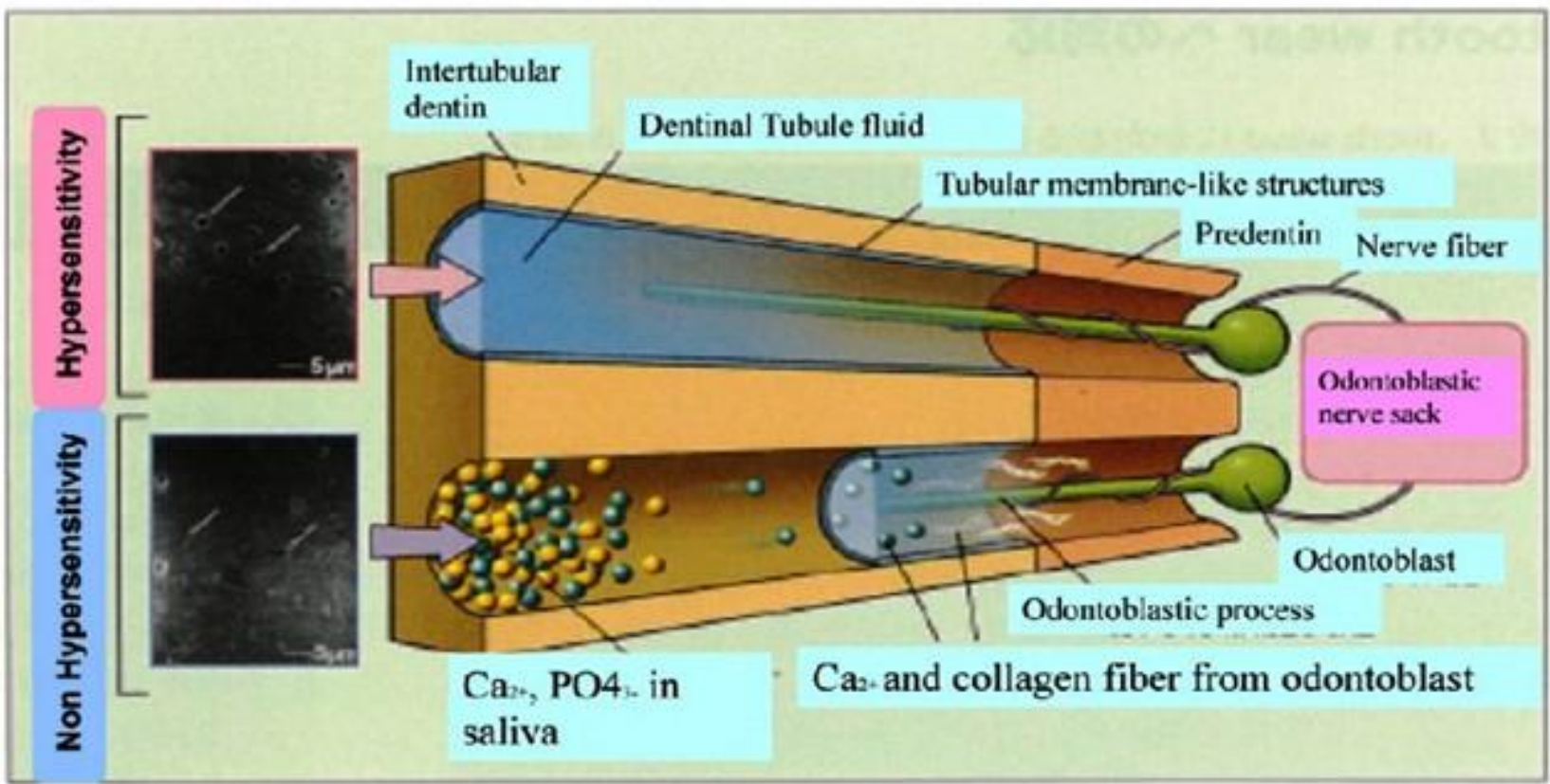

Fig. 3

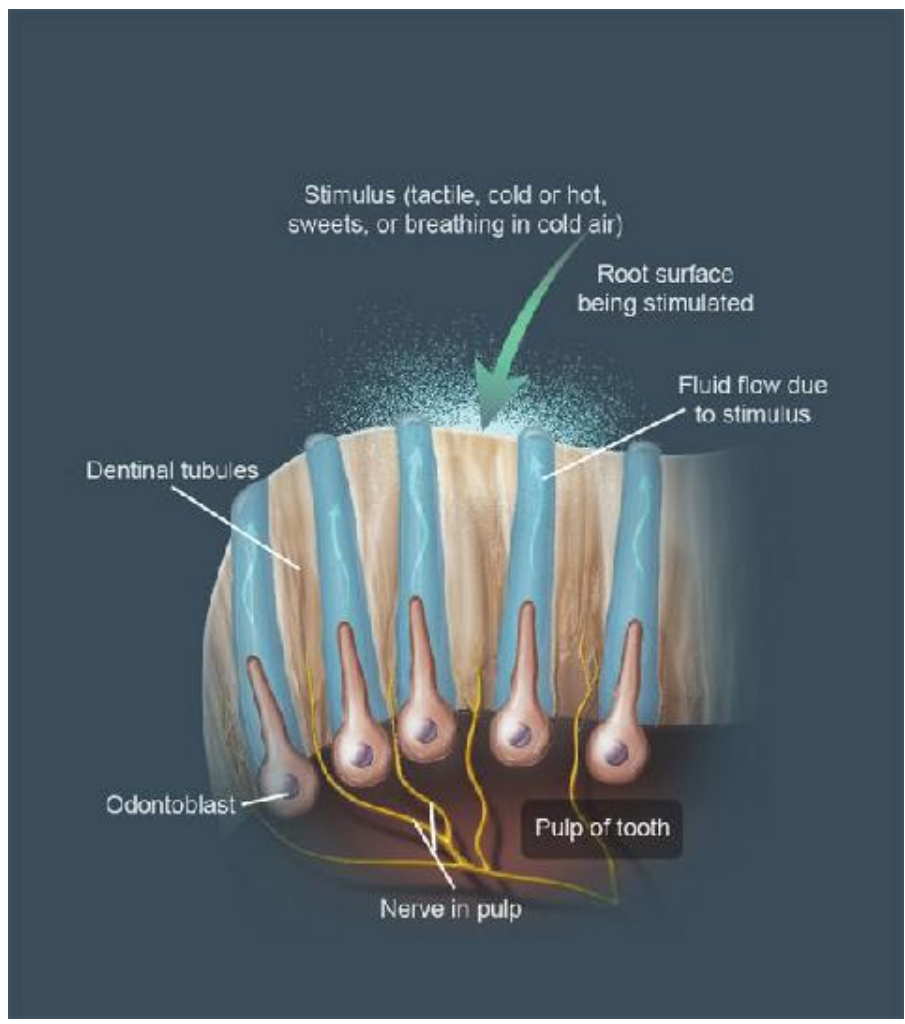

Fig. 4 


\section{REVIEW ARTICLE}

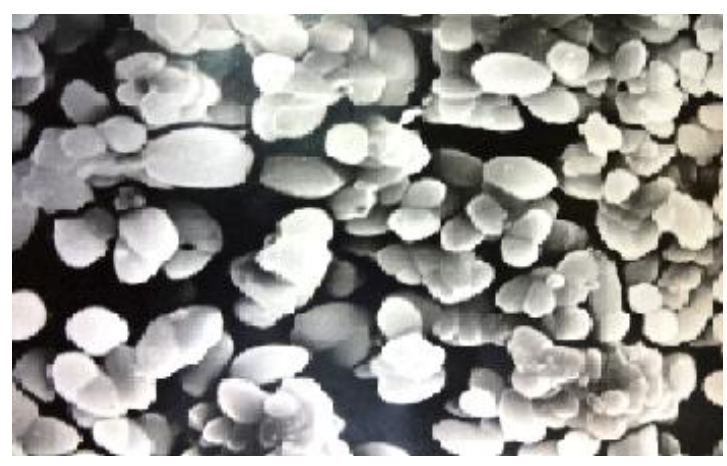

Fig. 5

\section{AUTHORS:}

1. Pradnya V. Bansode

2. Seema D. Pathak

3. Savita P. Wagh

\section{PARTICULARS OF CONTRIBUTORS:}

1. Professor and HOD, Department of Conservative Dentistry and Endodontics, Government Dental College \& Hospital, Aurangabad.

2. Assistant Professor, Department of Conservative Dentistry and Endodontics, Government Dental College \& Hospital, Aurangabad.
3. Dental Surgeon, Department of Conservative Dentistry and Endodontics, Government Dental College \& Hospital, Aurangabad.

\section{NAME ADDRESS EMAIL ID OF THE} CORRESPONDING AUTHOR:

Dr. Pradnya Bansode, 4, Sai Sakshi Residency, Plot No. 242, A Near Garden, Nandanvan Colony, Aurangabad.

Email-dr.pradnya_mds@rediffmail.com

Date of Submission: 01/08/2013.

Date of Peer Review: 02/08/2013.

Date of Acceptance: 13/08/2013.

Date of Publishing: 22/08/2013 